\title{
Características morfométricas, rendimentos de carcaça, filé, vísceras e resíduos em tilápias-do-nilo em diferentes faixas de peso ${ }^{1}$
}

\author{
Fredson Vieira e Silva ${ }^{2}$, Nilda Loiola de Almeida Franco e Sarmento ${ }^{3}$, Jodnes Sobreira Vieira ${ }^{4}$, \\ Antônio Jessey de Abreu Tessitore ${ }^{5}$, Laura Lúcia dos Santos Oliveira ${ }^{2}$, Edilson Paes Saraiva ${ }^{2}$
}

\author{
1 Projeto financiado pela Companhia de Desenvolvimento do Vale do São Francisco/CODEVASF. \\ 2 Departamento Ciências Agrárias/UNIMONTES. Bolsista de produtividade da FAPEMIG. \\ 3 Programa de Mestrado em Produção Vegetal da UNIMONTES. \\ ${ }^{4}$ Departamento Ciências Agrárias/UNIMONTES. \\ ${ }^{5}$ CODEVASF.
}

RESUMO - Objetivou-se com este trabalho avaliar o efeito do peso sobre as relações das medidas de comprimento padrão de tilápias-do-nilo (Oreochromis niloticus). Avaliaram-se as medidas: comprimento da cabeça; altura do corpo, medida à frente do $1^{\circ}$ raio das nadadeiras peitoral, dorsal, anal e caudal; largura do corpo, medida à frente da inserção do $1^{\circ}$ raio das nadadeiras peitoral, dorsal e anal; perímetro, medido à frente da inserção do $1^{\circ}$ raio das nadadeiras peitoral, dorsal e anal; e menor circunferência do pedúnculo; e os rendimentos de carcaça, filé, cabeça, nadadeira, pele, vísceras e resíduos. Foram utilizados 80 tilápias, em delineamento inteiramente casualizado com quatro tratamentos (faixas de peso 250 a 300 g; 350 a 400 g; 450 a 500 g, 550 a 600 g) e 20 repetições, considerando o peixe a unidade experimental. As menores variações nas características morfométricas foram observadas na faixa de peso de 450 a $600 \mathrm{~g}$ em comparação à faixa de 250 a $400 \mathrm{~g}$. Nas faixas de peso 250 a 300 g, 350 a 400 g, 450 a 500 g e 550 a 600 g, não houve diferenças nos rendimentos de carcaça e filé e nas porcentagens de cabeça, nadadeira, pele e resíduos. A porcentagem de vísceras na faixa de peso 550 a $600 \mathrm{~g}$ (10,98\%) foi significativamente inferior à obtida nas faixas de 250 a 300 g (12,77\%), 350 a 400 g (13,26\%) e 450 a 500 g (12,69\%). O peso do filé na faixa de peso 350 a $400 \mathrm{~g}$ (260,50 g) foi superior ao da faixa de 250 a 300 g (239,95 g). Não houve diferença no peso do filé entre as faixas de peso 450 a 500 g $(284,20$ g) e 550 a 600 g $(292,80$ g). As faixas de peso de 450 a 500 g e 550 a 600 g apresentaram valores superiores de peso do filé em comparação às de 250 a 300 g e 350 a 400 g. Considerando o peso do filé, o peso ideal de abate da tilápia é de 450 a 500 g no intervalo de peso de 250 a 600 g.

Palavras-chave: peso absoluto do filé, processamento do pescado, produção de pescado

\section{Morphometric characteristics, carcass, filet, viscera and residues in different weight categories of the Nile tilapia}

\begin{abstract}
The objective of this study was to evaluate the effect of weight categories on the relation of the measures of standard length; length of the head; height of the body measured at the front of the $1^{\circ}$ ray of pectoral, dorsal, anal and tail fins; width of the body measured at the front of the insertion of $1^{\circ}$ ray of pectoral, dorsal and anal fins; perimeter measured at the front of the insertion of the $1^{\circ}$ ray of pectoral, dorsal and anal fins and minor circumference of the peduncle and yield of carcass, filet, head, fin, skin, viscera and residues of the Nile tilapia (Oreochromis niloticus). Eighty tilapia were used, in a completely randomized design, with four treatments (weight categories 250 the $300 \mathrm{~g}$; 350 the $400 \mathrm{~g}$; 450 the $500 \mathrm{~g}$ and 550 the $600 \mathrm{~g}$ ) and 20 replications. Each fish was considered an experimental unit. The Nile tilapia presented minors variations in the morphometric characteristics in the weight category 450 to $600 \mathrm{~g}$, when compared with the category 250 to $400 \mathrm{~g}$. The weight category 250 the $300 \mathrm{~g}, 350$ the $400 \mathrm{~g}, 450$ the $500 \mathrm{~g}$ and 550 the 600 g provided the same yield of carcass, filet and percentages of head, fin, skin and residues. The percentage of viscera in the 550 the $600 \mathrm{~g}$ category (10.98\%) was significantly lower when compared to 250 the $300 \mathrm{~g}(12.77 \%), 350$ the $400 \mathrm{~g}(13.26 \%)$ and 450 the $500 \mathrm{~g}$ (12.69\%). The weight of filet in the 350 the $400 \mathrm{~g}$ category $(260.50 \mathrm{~g})$ was higher than the 250 the $300 \mathrm{~g}$ category (239.95 g). There was no difference in the weight of fillet between the 450 the $500 \mathrm{~g}$ category (284.20 g) and 550 the $600 \mathrm{~g}(292.80 \mathrm{~g})$. The category 450 the $500 \mathrm{~g}$ and 550 the $600 \mathrm{~g}$ presented higher values of weight of fillet when compared to 250 the $300 \mathrm{~g}$ and 350 the $400 \mathrm{~g}$. According to the weight of fillet found, the ideal weight of slaughter of the tilapia is between 450 and $500 \mathrm{~g}$ in a range of $250-600 \mathrm{~g}$.
\end{abstract}

Key Words: absolute weight of filet, fish processing, production of fished 


\section{Introdução}

A piscicultura vem se destacando como alternativa de alimento de alto valor nutritivo, no entanto, a produtividade encontra-se aquém do ideal. Neste contexto, o estudo das espécies deve ser priorizado para melhoria do desempenho animal e de tecnologias que aperfeiçoem as atividades da indústria de processamento de pescado.

Entre as espécies de excelente cultivo, destaca-se a tilápia-do-nilo (Oreochomis niloticus), que apresenta carne branca de textura firme, sabor delicado e fácil filetagem, não possui espinhas em 'y' nem odor desagradável. Além disso, é fácil reprodução e obtenção de alevinos, em virtude da possibilidade de manipulação hormonal do sexo para obtenção dos machos, da aceitação de diversos alimentos; da capacidade de aproveitar alimentos naturais em viveiros; do excelente crescimento em cultivo intensivo; da grande rusticidade (manejo intenso e baixos níveis de oxigênio dissolvido) e da resistência a doenças (Kubitza, 2000).

No entanto, do ponto de vista tecnológico, é necessário pesquisar acerca do padrão ou tamanho ideal do peixe para o abate que promova melhor rendimento para a indústria e atendimento das exigências dos mercados consumidores mais sofisticados (Pinheiro et al., 2006). O rendimento de filé está relacionado ao peso bruto do peixe, cujos valores variam de $25,4 \%$ até valores próximos a $42 \%$ (ContrerasGuzmán, 1994). Além do peso bruto, as medidas morfométricas podem ser utilizadas na avaliação da qualidade de carcaça como critério de seleção em programas de melhoramento genético (Freato et al., 2005) e para obter resposta correlacionada no rendimento do filé (Rutten et al., 2004).

Estratégias alternativas para melhoria de rendimento de filé em peixes têm se concentrado principalmente no uso de medidas do corpo como critério de seleção relacionado ao rendimento do filé. A forma de um animal não é apenas direcionada ao seu peso, mas também ao rendimento do filé do peixe (Bosworth et al., 2001).

Assim, objetivou-se com este trabalho avaliar o efeito de faixas de peso sobre as características morfométricas e os rendimentos de carcaça, filé, cabeça, nadadeira, pele, vísceras e resíduos de tilápias-do-nilo (Oreochromis niloticus).

\section{Material e Métodos}

O trabalho foi realizado na Estação de Hidrogeologia e Piscicultura da Companhia de Desenvolvimento do Vale do São Francisco (CODEVASF), situada no município de Nova Porteirinha, Minas Gerais, no mês de novembro de 2006.
Foram utilizadas 40 tilápias-do-nilo (Oreochromis niloticus), linhagem tailandesa, de quatro faixas de peso. Cada peixe foi considerado uma unidade experimental, totalizando 40 unidades.

O delineamento experimental foi inteiramente casualizado, com quatro tratamentos (faixas de peso 250 a 300 g; 350 a 400 g; 450 a 500 g e 550 a 600 g) e 10 repetições por tratamento. No estudo, alevinos de mesmo lote, pesando em média $12 \mathrm{~g}$, foram estocados e cultivados em um viveiro de terra de $200 \mathrm{~m}^{2}$. Os peixes foram alimentados com ração comercial contendo os níveis de proteína bruta requeridos para cada fase do desenvolvimento, em quantidade adequada para a biomassa do tanque e a temperatura da água.

Os peixes foram capturados por meio de rede de arrasto e transportados em caixas de isopor até o tanque de depuração, localizado a $150 \mathrm{~m}$ do tanque de criação. Após jejum de 24 horas, foram transportados em caixas térmicas até o local do processamento. Os animais foram abatidos por meio de choque térmico a temperatura em torno de $0^{\circ} \mathrm{C}$ e tempo de aproximadamente 2 minutos.

Cada exemplar, depois de morto, foi submetido às medições de comprimento padrão, que compreende a extremidade anterior da cabeça e o menor perímetro do pedúnculo (inserção da nadadeira caudal); comprimento da cabeça, que compreende a extremidade anterior da cabeça e o bordo caudal do opérculo; altura do corpo medida à frente do $1^{\circ}$ raio das nadadeiras peitoral, dorsal e anal; largura do corpo medida à frente da inserção do $1^{\circ}$ raio das nadadeiras peitoral, dorsal e anal; perímetro medido à frente da inserção do $1^{\circ}$ raio das nadadeiras peitoral, dorsal, anal e na menor circunferência do pedúnculo.

Para determinação do rendimento no processamento, foram pesados as vísceras (todo o conteúdo da cavidade celomática, inclusive as gônadas e gorduras celomáticas), a pele e as escamas, o filé (carne livre da pele e ossos, isca e costelinha), a cabeça (seccionada do corpo na altura da junção com a coluna vertebral, incluindo brânquias), o resíduo da filetagem (isca e a costelinha) e as nadadeiras peitoral, dorsal, caudal e anal (a nadadeira anal foi seccionada à altura do perímetro do pedúnculo).

As medidas foram realizadas com fita métrica e paquímetro, graduados em milímetros (mm), utilizando-se balança digital de $10 \mathrm{~kg}$ de capacidade, com sensibilidade de $0,1 \mathrm{~g}$, além de faca, tesoura e alicate para a separação do filé.

Os dados de rendimento foram calculados em relação ao peso do exemplar. O processo de filetagem foi manual e realizado pela mesma pessoa, assim como os demais procedimentos de abate, de modo que cada pessoa, previamente treinada, executou determinada tarefa. 
Os dados de rendimentos de carcaça e filé, porcentagens de cabeça, nadadeira, pele, vísceras, resíduos e peso absoluto de filé e características morfométricas foram submetidos à análise de variância e suas médias comparadas pelo teste Tukey a 5\% de probabilidade (Ribeiro Jr., 2001).

\section{Resultados e Discussão}

As faixas de peso de 250 a $300 \mathrm{~g}, 350$ a $400 \mathrm{~g}, 450$ a $500 \mathrm{~g}$ e 550 a 600 g proporcionaram os mesmos rendimentos de carcaça, cuja média foi de 59,10\% (Tabela 1). Maior rendimento reflete maior eficiência em ganho de carcaça e tem maior valor econômico que os demais componentes que não fazem parte da carcaça. No entanto, mesmo os animais da faixa de peso 250 a $300 \mathrm{~g}$ não foram inferiores, quanto a este parâmetro, àqueles das demais faixas de peso, portanto, tiveram mesma eficiência para ganho de carcaça.

Esses resultados apresentados são superiores aos relatados por Souza et al. (1999), que verificaram rendimento de carcaça de 53,27 a 56,49\% em tilápias de 250 a 450 g, e por Clement \& Lovell (1994), que obtiveram valor de 51\% de rendimento de carcaça. Entretanto, Souza et al. (1999) observaram que peixes mais pesados apresentaram maior rendimento de carcaça. Segundo Contreras-Guzmán(1994), a carcaça representa, em média, $62,6 \%$ do peso dos peixes. Assim, a avaliação do rendimento de carcaças é importante para melhoria da eficiência dos sistemas de produção, e a eficácia da avaliação aumenta quando comparados simultaneamente outros parâmetros que determinam e caracterizam o crescimento e desenvolvimento animal.

Não houve diferença significativa $(\mathrm{P}>0,05)$ no rendimento de filé, em média 34,18\%, entre as faixas de peso 250 a 300 g, 350 a 400 g, 450 a 500 g e 550 a 600 g. Clement \& Lovell (1994) relataram rendimento de filé de $25,4 \%$ para tilápia com peso médio de $585 \mathrm{~g}$. Resultado semelhante ao deste estudo foi descrito por Macedo-Viegas et al. (1997), que encontraram, em peixes pesando de 250 a $450 \mathrm{~g}$, valores de 32,15 a 40,39\%. Entretanto, esses autores observaram que peixes mais pesados apresentam maior rendimento de filé.
Da mesma forma, Souza et al. (1998) relataram influência do peso de abate no rendimento de filé em tilápias-do-nilo.

Assim, mesmo em peixes de 250 a $600 \mathrm{~g}$, as relações entre pesos de carcaça e filé e peso vivo não estiveram relacionadas ao peso absoluto dos peixes. Portanto, peixes nas faixas 250 a 300 g, 350 a 400 g, 450 a 500 g e 550 a 600 g não apresentaram alterações em seus rendimentos de carcaça e filé, o que difere dos relatos de Lawrie (2005) em estudo com outras espécies. Souza et al. (2005) relataram que filés de peixes das faixas de peso 601 a 700 g e 701 a 800 g apresentaram melhor rendimento em relação àqueles peixes na faixa de 501 a 600 g. No entanto, segundo Rasmussen \& Ostenfeld (2000), o rendimento de filé não é afetado pelo crescimento do peixe, mas a espécie pode ter efeito sobre essa variável, assim como sobre o grau de mecanização, o método de filetagem e a destreza do filetador. Resultado semelhante foi obtido por Santos et al. (2007 a), que verificaram que o crescimento das partes do corpo de tilápias das linhagens Chitralada foi proporcional ao aumento do peso corporal e que esse aumento não alterou o rendimento desses constituintes.

O peso absoluto de filé na faixa de peso 350 a $400 \mathrm{~g}$ foi superior ao da faixa de 250 a $300 \mathrm{~g}$. Não houve diferença no peso absoluto de filé entre as faixas de peso 450 a $500 \mathrm{~g}$ e 550 a 600 g. As faixas de peso 450 a 500 g e 550 a 600 g apresetaram valores superiores de peso de filé em comparação às de 250 a 300 g e 350 a 400 g. Segundo Souza et al. (2005), para a defumação, é interessante um filé de maior tamanho, peso e espessura, pois a desidratação ocorreria mais superficialmente durante o processo e não seria excessiva para não afetar a textura do produto.

Como peixes de menor peso têm melhor conversão alimentar, a faixa de peso 450 a 500 g sobressai-se em comparação à de 550 a 600 g, uma vez que, apesar do mesmo peso de filé de ambas as faixas, o peso vivo da faixa 450 a $500 \mathrm{~g}$ foi menor.

As porcentagens de cabeça, nadadeira, pele e resíduos não diferiram entre as faixas de peso estudadas (Tabela 2). Bosworth et al. (1998) relataram que menor proporção da cabeça, ossos e nadadeiras nos peixes com forma corporal volumosa está relacionada a elevados rendimentos de filé.

Tabela 1 - Rendimentos de carcaça, filé e peso absoluto da carcaça e do filé de tilápias-do-nilo

\begin{tabular}{lccrrrr}
\hline Variável & \multicolumn{4}{c}{ Faixa de peso de abate } & \multicolumn{2}{c}{ Média } \\
\cline { 2 - 5 } & 250 a $300 \mathrm{~g}$ & $350 \mathrm{a} 400 \mathrm{~g}$ & $450 \mathrm{a} 500 \mathrm{~g}$ & 550 a $600 \mathrm{~g}$ & \\
\hline Peso da carcaça (g) & $241,0 \mathrm{a}$ & $318,0 \mathrm{~b}$ & $414,0 \mathrm{c}$ & $497,0 \mathrm{~d}$ & $367,55 \pm 100$ \\
Rendimento de carcaça (\%) & $59,39 \mathrm{a}$ & $58,22 \mathrm{a}$ & $58,20 \mathrm{a}$ & $60,56 \mathrm{a}$ & $59,10 \pm 2,3$ & 3,59 \\
Peso de filé (g) & $239,95 \mathrm{a}$ & $260,50 \mathrm{~b}$ & $284,20 \mathrm{c}$ & $292,80 \mathrm{c}$ & $269,36 \pm 22$ & 3,10 \\
Rendimento de filé (\%) & $33,22 \mathrm{a}$ & $34,26 \mathrm{a}$ & $33,83 \mathrm{a}$ & $35,41 \mathrm{a}$ & $34,18 \pm 2,3$ \\
\hline
\end{tabular}

Letras diferentes na mesma linha diferem entre si pelo teste Tukey $(\mathrm{P}<0,05)$; CV = coeficiente de variação. 
As vísceras na faixa de peso 550 a $600 \mathrm{~g}$ foram significativamente inferiores $(\mathrm{P}<0,05)$ em comparação às de $250 \mathrm{a}$ 300 g, 350 a 400 g e 450 a 500 g (Tabela 2). Os dados obtidos sugerem que a adição de nova faixa de peso (650 a 700 g) pode levar a um maior rendimento de carcaça, em decorrência de uma suposta diminuição da porcentagem das vísceras com o aumento do peso. No entanto, a adição de uma nova faixa e o possível aumento de rendimento não asseguram maior eficiência produtiva, em razão do aumento da conversão de alimentos em carne.

As relações dos dados morfométricos não diferiram $(\mathrm{P}>0,05)$ nas faixas de peso de 250 a $600 \mathrm{~g}$ (Tabela 3). Leonhardt et al. (2006), avaliando diferentes linhagens de tilápias-do-nilo, encontraram valores semelhantes de comprimento do tronco/comprimento padrão e comprimento da cabeça/comprimento padrão. De acordo Boscolo et al. (2001), as relações largura/comprimento e largura/altura representam a conformação do filé e indicam que os peixes são mais robustos quando as relações são mais elevadas. Entretanto, relações morfométricas e suas curvas de crescimento em peixes, mais especificamente em linhagens de tilápias, não têm sido estudadas (Santos et al., 2007a). Santos et al. (2007b) concluíram que carcaça com menores relações altura/largura e maior perímetro/altura pode ser indicativa de maior rendimento de partes comestíveis.
Neste contexto, os valores, nas faixas 250 a 300 g, 350 a 400 g, 450 a 500 g e 550 a 600 g comprovaram que não houve alterações $(\mathrm{P}>0,05)$ nas principais relações que indicam rendimento de partes comestíveis e mudança na conformação do filé.

As características morfométricas altura do corpo medida à frente do $1^{\circ}$ raio das nadadeiras dorsal e anal e perímetro medido à frente do $1^{\circ}$ raio da nadadeira anal aumentaram $(\mathrm{P}<0,05)$ em todas as faixas de peso analisadas. O comprimento padrão, comprimento de cabeça, altura do corpo medida à frente do $1^{\circ}$ raio da nadadeira peitoral, largura do corpo medida à frente do $1^{\circ}$ raio das nadadeiras peitoral, dorsal e anal, perímetro medido à frente do $1^{\circ}$ raio da nadadeiras peitoral e dorsal e menor circunferência do pendúnculo, não diferiram $(\mathrm{P}>0,05)$ nas faixas de peso 450 a 500 g e 550 a $600 \mathrm{~g}$ (Tabela 4), ou seja, quando o peixe atinge o peso de 450 g essas variáveis não sofreram alterações significativas em suas medidas. Assim, as alterações morfométricas na tilápia diminuíram, na maioria dos parâmetros avaliados, quando os peixes atingiram a faixa de peso 450 a $500 \mathrm{~g}$ e foram mais acentuadas nas faixas de peso 250 a 300 g e 350 a 400 g. As considerações são de suma importância para a indústria de pescado, pois as medidas estão altamente correlacionadas ao peso do filé, corte comercial de maior valor econômico da carcaça dos peixes.

Tabela 2 - Valores médios de cabeça, nadadeiras, pele, vísceras e resíduos de tilápias-do-nilo

\begin{tabular}{lrrrrr}
\hline Variável & \multicolumn{4}{c}{ Faixa de peso de abate } & Média \\
\cline { 2 - 4 } & 250 a $300 \mathrm{~g}$ & 350 a $400 \mathrm{~g}$ & 450 a $500 \mathrm{~g}$ & 550 a $600 \mathrm{~g}$ \\
\hline Cabeça (\%) & $21,78 \mathrm{a}$ & $21,29 \mathrm{a}$ & $22,40 \mathrm{a}$ & $20,76 \mathrm{a}$ & $21,56 \pm 1,76$ \\
Nadadeiras (\%) & $3,61 \mathrm{a}$ & $3,90 \mathrm{a}$ & $4,08 \mathrm{a}$ & $4,17 \mathrm{a}$ & $3,94 \pm 0,58$ \\
Pele (\%) & $6,31 \mathrm{a}$ & $5,62 \mathrm{a}$ & $6,08 \mathrm{a}$ & $5,77 \mathrm{a}$ & $5,95 \pm 0,77$ \\
Vísceras (\%) & $12,77 \mathrm{a}$ & $13,26 \mathrm{a}$ & $12,69 \mathrm{a}$ & $10,98 \mathrm{~b}$ & $12,43 \pm 1,61$ \\
Resíduos (\%) & $9,55 \mathrm{a}$ & $9,76 \mathrm{a}$ & $10,06 \mathrm{a}$ & $10,65 \mathrm{a}$ & $10,01 \pm 1,37$ \\
\hline
\end{tabular}

Letras diferentes na mesma linha diferem $(\mathrm{P}<0,05)$ entre si pelo teste Tukey; $\mathrm{CV}=$ coeficiente de variação.

Tabela 3 - Características morfométricas de tilápias-do-nilo abatidas em diversas faixas de peso

\begin{tabular}{|c|c|c|c|c|c|c|}
\hline \multirow[t]{2}{*}{ Variável } & \multicolumn{4}{|c|}{ Faixa de peso de abate } & \multirow[t]{2}{*}{ Média } & \multirow[t]{2}{*}{ CV (\%) } \\
\hline & 250 a $300 \mathrm{~g}$ & 350 a $400 \mathrm{~g}$ & 450 a $500 \mathrm{~g}$ & 550 a $600 \mathrm{~g}$ & & \\
\hline Comprimento cabeça/comprimento padrão & $0,30 \mathrm{a}$ & $0,30 \mathrm{a}$ & $0,29 a$ & $0,29 a$ & $0,29 \pm 0,01$ & 4,75 \\
\hline Comprimento cabeça/altura cabeça & $0,81 \mathrm{a}$ & 0,80 a & $0,79 a$ & $0,78 \mathrm{a}$ & $0,79 \pm 0,03$ & 3,71 \\
\hline Comprimento padrão/comprimento total & $0,87 a$ & $0,85 a$ & $0,85 a$ & $0,84 \mathrm{a}$ & $0,85 \pm 0,10$ & 12,13 \\
\hline Largura corpo/altura corpo & $0,48 \mathrm{a}$ & $0,46 \mathrm{a}$ & $0,46 a$ & $0,51 \mathrm{a}$ & $0,48 \pm 0,08$ & 16,36 \\
\hline Largura corpo/comprimento tronco & $0,25 \mathrm{a}$ & $0,25 a$ & $0,24 a$ & $0,27 \mathrm{a}$ & $0,25 \pm 0,04$ & 16,19 \\
\hline Altura corpo/comprimento tronco & $0,53 a$ & $0,54 a$ & $0,52 \mathrm{a}$ & $0,51 \mathrm{a}$ & $0,52 \pm 0,03$ & 6,59 \\
\hline Comprimento tronco/comprimento padrão & $0,70 \mathrm{a}$ & $0,70 \mathrm{a}$ & $0,71 \mathrm{a}$ & $0,71 \mathrm{a}$ & $0,71 \pm 0,01$ & 1,97 \\
\hline Altura corpo/comprimento padrão & $0,37 \mathrm{a}$ & $0,38 \mathrm{a}$ & $0,37 a$ & $0,37 a$ & $0,37 \pm 0,02$ & 4,87 \\
\hline Altura cabeça/comprimento cabeça & $1,23 a$ & $1,26 a$ & $1,27 \mathrm{a}$ & $1,28 \mathrm{a}$ & $1,26 \pm 0,05$ & 3,64 \\
\hline Perímetro corpo/comprimento padrão & $0,94 \mathrm{a}$ & 0,93 a & $0,92 \mathrm{a}$ & $0,91 \mathrm{a}$ & $0,93 \pm 0,04$ & 4,61 \\
\hline
\end{tabular}

Letras diferentes na mesma linha diferem $(\mathrm{P}<0,05)$ entre si pelo teste Tukey; CV = coeficiente de variação. 
Tabela 4 - Características morfométricas de tilápias-do-nilo em diversas faixas de peso

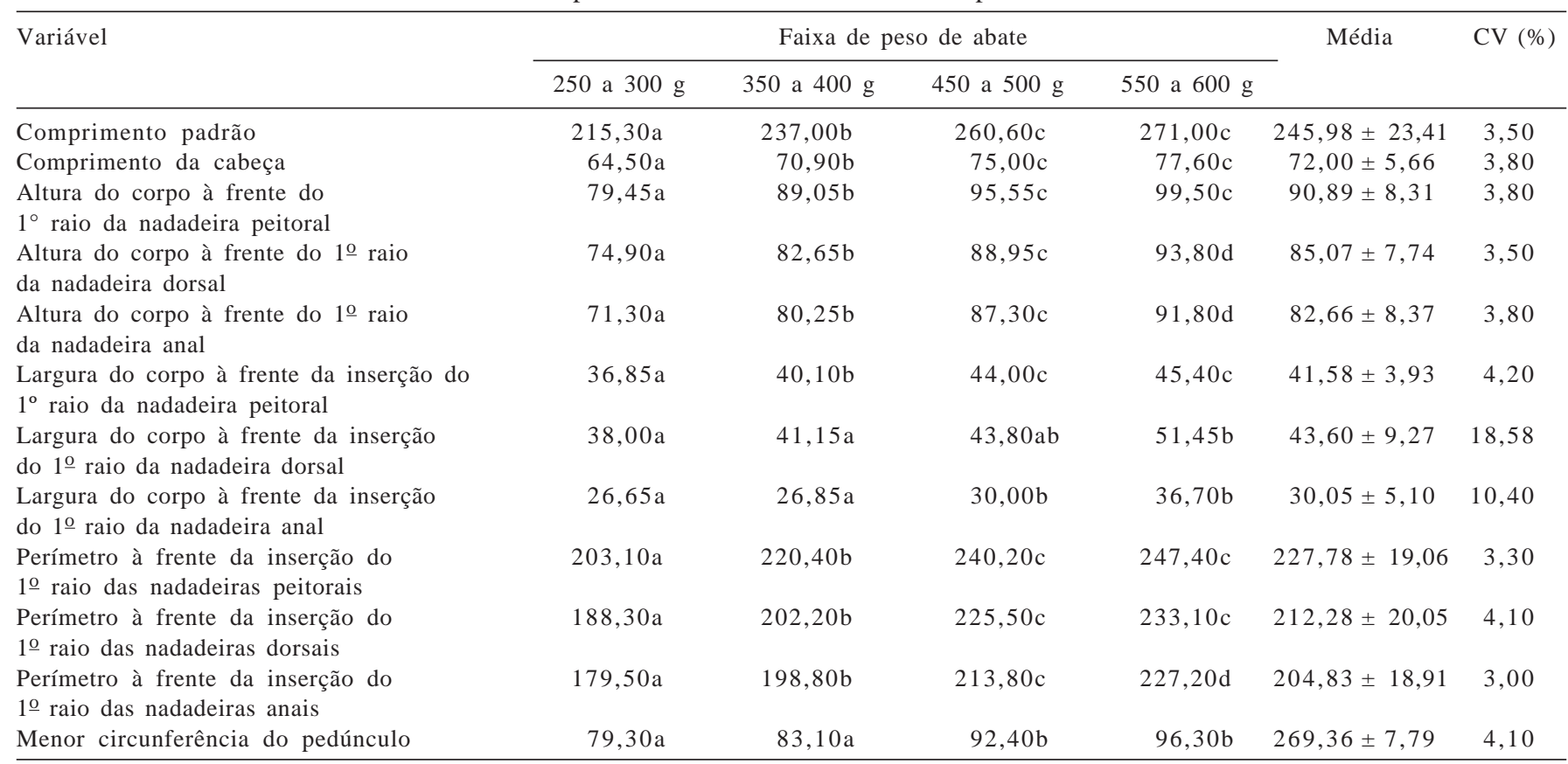

Letras diferentes na mesma linha diferem $(\mathrm{P}<0,05)$ entre si pelo teste Tukey; $C V=$ coeficiente de variação.

\section{Conclusões}

Em tilápias-do-nilo, as menores variações nas características morfométricas ocorrem na faixa de peso de 450 a 600 g em relação à faixa de 250 a 400 g. O crescimento das partes constituintes do corpo de tilápias-do-nilo da linhagem tailandesa é proporcional ao aumento do peso corporal. Não houve diferença nos rendimentos de carcaça e filé na faixa de peso de 250 a $600 \mathrm{~g}$. De acordo com o peso absoluto de filé encontrado, o peso ideal de abate da tilápia é de 450 a $500 \mathrm{~g}$.

\section{Agradecimentos}

Aos funcionários da Estação de Hidrogeologia e Piscicultura da CODEVASF, situada no município de Nova Porteirinha, Minas Gerais.

\section{Literatura Citada}

BOSCOLO, W.R.; HAYASHI, C.; SOARES, C.M. et al. Desempenho e características de carcaça de machos revertidos de tilápia do Nilo (Oreochromis niloticos), linhagens tailandesa e comum, nas fases inicial e de crescimento. Revista Brasileira de Zootecnia, v.30, n.5, p.1391-1396, 2001.

BOSWORTH, B.G.; LIBEY, G.S.; NOTTER, D.R. Relationships among total weight, body shape, visceral components, and fillet traits in Palmetto Bass (Striped Bass female Morone sascatilis $\mathrm{x}$ White Bass male M. chrysops) and Paradise Bass (Striped Bass female M. saxatilis x Yellow Bass M. minissippiensis). Journal of the World Aquaculture Society, v.29, n.1, p.40-50, 1998.
BOSWORTH, B.G; HOLLAND, M.; BRAZIL, B.L. Evaluation of ultrasound imagery and body shape to predict carcass and fillet yeild in farm-raised catfhis. Journal of Animal Science, v.79, n.6, p.1483-1490, 2001.

CLEMENT, S.; LOVELL, R.T. Comparison of processing yield and nutrient composition of culture Nile tilapia (Oreochromis niloticus) and channel catfish (Ictalurus punctatus). Aquaculture, v.119, p.299-310, 1994.

CONTRERAS-GUZMÁN, E.S. Bioquímica de pescados e derivados. Jaboticabal: FUNEP, 1994. 409p.

FREATO, T.A.; FREITAS, R.T.F.; SANTOS, V.B. et al. Efeito do peso de abate nos rendimentos do processamento da piracanjuba (Brycon orbignyanus, Valenciennes, 1849). Ciência e Agrotecnologia, v.29, n.3, p.676-682, 2005.

KUBITZA F. Tecnologia e planejamento na produção comercial. 1.ed. São Paulo: Jundiaí, 2000. 285p.

LAWRIE, R.A. Ciência da carne. 6.ed. Porto Alegre: Artmed, 2005. 384p.

LEONHARDT, J.H.; CAETANO FILHO, M.; FROSSARD, H. et al. Características morfométricas, rendimento e composição do filé de tilápia do Nilo, Oreochromis niloticus, da linhagem tailandesa, local e do cruzamento de ambas. Semina: Ciências Agrárias, v.27, n.1, p.125-132, 2006.

MACEDO-VIEGAS, E.M.; SOUZA, M.L.R.; KRONKA, S.N. Estudo da carcaça de tilápia do Nilo (Oreochromis niloticus), em quatro categorias de peso. Revista UNIMAR, v.19, n.3, p.863-870, 1997.

PINHEIRO, L.M.S.; MARTINS, R.T.; PINHEIRO, L.A.S. et al. Rendimento industrial de filetagem da tilápia tailandesa (Oreochromis ssp). Arquivo Brasileiro de Medicina Veterinária e Zootecnia, v.58, n.2, p.257-262, 2006.

RASMUSSEN, R.S.; OSTENFELD, T.H. Effect of growth rate on quality traits and feed utilization of rainbow trout (Oncorhyncus mykiss) and brook trout (Salvelinus fontinalis). Aquaculture, v.184, p.327-337, 2000.

RIBEIRO JR., J.I. Sistema de análises estatísticas e genéticas - SAEG. Viçosa, MG: 2001. 301p.

RUTTEN, M.J.M.; BOVENHUIS, H.; KOMEN, H. Modeling fillet traits based on body measuraments in three Nile tilapia satrains 
(Oreochromis niloticus L.). Aquaculture, v.231, n.1-4, p.113-122, 2004.

SANTOS, V.B.; FREITAS, R.T.F.; SILVA, F.F. et al. Avaliação de curvas de crescimento morfométrico de linhagens de tilápia do Nilo (Oreochromis niloticus). Ciência e Agrotecnologia, v.31, n.5, p.1486-1492, 2007a.

SANTOS, V.B.; FREITAS, R.T.F.; LOGATO, P.V.R. et al. Rendimento do processamento de linhagens de tilápias (Oreochromis niloticus) em função do peso corporal. Ciência e Agrotecnologia, v.31, n.2, p.554-562, 2007b.

SOUZA, M.L.R.; CASTAGNOLLI, N.; KRONKA, S.N. Influência das densidades de estocagem e sistema de aeração sobre o peso e características de carcaça da tilápia do Nilo (Oreochromis niloticus). Acta Scientiarum, v.20, n.3, p.387-393, 1998.

SOUZA, M.L.R.; MACEDO-VIEGAS E.M.; KRONKA, S.N. Influência do método de filetagem e categorias de peso sobre rendimento de carcaça, filé e pele da tilápia do Nilo (Oreochromis niloticus). Revista Brasileira de Zootecnia, v.28, n.1, p.1-6, 1999.

SOUZA, M.L.R.; MACEDO-VIEGAS E.M.; SOBRAL, P.J.A. KRONKA, S.N. Efeito do peso de tilápia do Nilo (Oreochromis niloticus) sobre o rendimento e a qualidade de seus filés defumados com e sem pele. Ciência e Tecnologia de Alimentos, v.25, n.1, p.51-59, 2005. 